\title{
L'EBSI, les compétences disciplinaires et professionnelles et la question de la relève
}

\author{
The EBSI, Subject-Area and Professional Competencies, and \\ New Graduates
}

\section{La Escuela de Biblioteconomía y Ciencias de la Información - EBSI: la competencia disciplinaria y profesional y la cuestión del relevo}

\section{Marcel Lajeunesse et Carol Couture}

Volume 50, numéro 2, avril-juin 2004

URI : https://id.erudit.org/iderudit/1030081ar

DOI : https://doi.org/10.7202/1030081ar

Aller au sommaire du numéro

Éditeur(s)

Association pour l'avancement des sciences et des techniques de la documentation (ASTED)

ISSN

0315-2340 (imprimé)

2291-8949 (numérique)

Découvrir la revue

Citer cet article

Lajeunesse, M. \& Couture, C. (2004). L’EBSI, les compétences disciplinaires et professionnelles et la question de la relève. Documentation et bibliothèques, 50(2), 169-174. https://doi.org/10.7202/1030081ar

\section{Résumé de l'article}

Il est normal que les associations professionnelles qui regroupent des professionnels diplômés d'une même discipline ainsi que les écoles universitaires qui forment les futurs professionnels s'interrogent périodiquement sur leur mission respective, tant au plan de la situation actuelle que sur un avenir prévisible. Après des décennies de compressions budgétaires, de non-embauche des jeunes diplômés et de promotion interne sans recours à des recrutements de candidats de l'extérieur, les organismes documentaires, bibliothèques et services d'archives du secteur public et parapublic, s'interrogent actuellement avec acuité sur leur avenir, en posant le problème des compétences disciplinaires et professionnelles par rapport à l'urgence de la relève.
Tous droits réservés $(\subset)$ Association pour l'avancement des sciences et des techniques de la documentation (ASTED), 2004
Ce document est protégé par la loi sur le droit d'auteur. L'utilisation des services d'Érudit (y compris la reproduction) est assujettie à sa politique d'utilisation que vous pouvez consulter en ligne. 


\section{L'EBSI, les compétences disciplinaires et professionnelles et la question de la relève}

MARCEL LAJEUNESSE

Professeur titulaire

EBSI, Université de Montréal

marcel.lajeunesse@umontreal.ca

\author{
CAROL COUTURE \\ Directeur et professeur titulaire \\ EBSI, Université de Montréal \\ Carol.couture@umontreal.ca
}

\section{RÉSUMÉ | ABSTRACTS | RESUMEN \\ Il est normal que les associations professionnelles qui regroupent des professionnels diplômés d'une même discipline ainsi que les écoles universitaires qui forment les futurs professionnels sinter- rogent périodiquement sur leur mission respective, tant au plan de la situation actuelle que sur un avenir prévisible. Après des décennies de compressions budgétaires, de non-embauche de jeu- nes diplômés et de promotion interne sans recours à des recrute- ments de candidats de l'extérieur, les organismes documentaires, bibliothèques et services d'archives du secteur public et parapu- blic, s'interrogent actuellement avec acuité sur leur avenir, en posant le problème des compétences disciplinaires et profession- nelles par rapport à l'urgence de la relève.}

The EBSI, Subject-Area and Professional Competencies, and New Graduates

From time to time, it is expected for professional associations that represent the graduates of a given discipline, as well as the universities that train these professionals, to assess their missions as well as their prospects. Following several years of budgetary restraint, the non-hiring of new graduates and internal promotion without external recruitment, the library and archives services of the public and parapublic sectors are evaluating the future in the light of the subject-area and professional competencies required for the urgent renewal of human resources.

\section{La Escuela de Biblioteconomía y Ciencias de la Información - EBSI: la competencia disciplinaria y profesional y la cuestión del relevo}

Es normal que las asociaciones profesionales que agrupan diplomados de una misma disciplina y las escuelas universitarias que forman futuros profesionales se interroguen periódicamente sobre sus respectivas misiones, tanto con respecto a la situación actual como sobre el porvenir previsible. Después de décadas de reducciones presupuestarias, de no emplear jóvenes diplomados $y$ de promociones internas sin posibilidades de reclutamientos de candidatos del exterior, los organismos de documentación, bibliotecas y servicios de archivos de los sectores público y parapúblico se interrogan actualmente con preocupación sobre su porvenir, planteando el problema de la competencia en las distintas disciplinas y profesiones en vista de la urgencia del relevo.

\section{DU PROBLÈME DE LA RELÈVE DANS NOS ORGANISATIONS}

L

es universités canadiennes doivent embaucher, au cours de la décennie 2000-2010, de 30000 à 40000 nouveaux professeurs pour remplacer ceux qui partiront à la retraite et pour rencontrer les nouveaux besoins en enseignement et en recherche (AUCC 2002). Or, les universités canadiennes ne forment que quelque 4000 docteurs par an et environ 30 à $35 \%$ seulement de ces nouveaux Ph.D. feront une carrière de professeur d'université. La problématique $\mathrm{du}$ renouvellement du corps professoral est posée d'une manière similaire aux États-Unis et en Europe. De plus, la situation du début du XXI ${ }^{\mathrm{e}}$ siècle est bien différente de celle des années 70, au moment de l'embauche de nombreux professeurs européens dans nos universités. Elle s'est enrichie de nombreuses nouvelles universités et s'est dotée de laboratoires de recherche performants. La nécessaire relève dans les universités canadiennes crée une compétition très vive entre établissements, et les concours de recrutement, de plus en plus internationaux, doivent bonifier les conditions offertes aux candidats. En considérant les réalités que vivent les universités, le gouvernement du Canada a même assoupli ses règles concernant les candidatures d'étrangers et leur venue dans les universités canadiennes. Quant au gouvernement du Québec, il a exonéré d'impôts, pour les cinq premières années, les nouveaux professeurs de certaines disciplines venus de l'étranger.

William Curran, de l'Université Concordia, a posé avec beaucoup de justesse le problème du renouvellement du personnel professionnel dans les bibliothèques universitaires du Québec (Curran 2002). Les faits parlent d'eux-mêmes. La Corporation des bibliothécaires professionnels du Québec nous a informés que plus de $20 \%$ de ses membres actuels ont déjà atteint l'âge de 55 ans et qu'il est vraisemblable que ceux-ci seront à la retraite d'ici cinq à huit ans. Une autre étude canadienne nous apprend que $50 \%$ des bibliothécaires œuvrant dans les bibliothèques universitaires et de recherche auront quitté le marché du travail d'ici 15 ans. Le rapport annuel des bibliothèques de l'Université McGill de 2002 révèle que $55 \%$ de son personnel professionnel a plus de 50 ans. Des sondages effectués dans d'autres bibliothèques universitaires du Québec, notamment dans les Universités 
Laval et de Montréal, dévoilent des chiffres similaires, quand ils ne sont pas plus élevés. Ce problème du remplacement du personnel professionnel n'est pas que québécois, il préoccupe grandement les associations professionnelles de bibliothécaires nordaméricaines (Law 2000 et Hernon-Powell-Young 2001).

La question de la relève n'est pas limitée au monde universitaire. Les écoles de niveau primaire et secondaire, les collèges d'enseignement général et professionnel, la fonction publique, qui ont fait beaucoup d'embauches au tournant des années 70 , sont confrontés au même défi du remplacement d'un nombre élevé de leurs membres (professeurs, professionnels, fonctionnaires). La gestion du remplacement et de la relève fait partie de la planification des ressources humaines, il est normal que toute organisation la prenne en compte. La particularité de la situation présente concerne le taux de remplacement: il est élevé, et dans beaucoup de cas, il est anormalement élevé. Avec le départ massif de leurs employés, les institutions se privent d'une expertise précieuse et d'une mémoire indispensable, en plus de l'absence d'un mentorat nécessaire aux nouvelles recrues.

Revenons aux bibliothèques universitaires dont l'état des besoins imminents de personnel professionnel est bien documenté. De 1980 à 1999, le nombre de bibliothécaires professionnels dans les universités québécoises est passé de 486 à 337, la diminution étant plus significative de 1995 à 1999 (CRÉPUQ 1999). Cette réduction des effectifs est le résultat des coupures budgétaires combinées avec des départs à la retraite dans le cadre de programmes prévus entre 1995 et 1999. Pendant des années, il n'y a pas eu de nouvelles embauches, et de nombreux postes de cadres intermédiaires ont été abolis. Il s'est opéré un allègement ou "aplatissement» des organigrammes, selon la terminologie des relations industrielles. Les possibilités d'avancement ou de promotion pour le personnel professionnel en poste étaient minces au cours de cette période. Pourtant, c'est par l'exercice de fonction de coordination et de gestion intermédiaire que les professionnels peuvent faire la démonstration de leur capacité et de leur talent de gestionnaire, et qu'ils peuvent espérer accéder à des postes supérieurs de direction de bibliothèques. Les bibliothèques devront affronter, au cours des prochaines années, un double défi. D'abord, pour contrer la diminution du nombre de professionnels, on devra recruter un grand nombre de nouveaux professionnels, ensuite, pour pallier les carences de la dernière décennie, il incom- bera de former et de recruter des gestionnaires à tous les niveaux de l'organisation.

\section{LES COMPÉTENCES DES DIPLÔMÉS DES ÉCOLES DE SCIENCES DE L'INFORMATION}

Il est dans l'ordre des choses qu'une école à finalité professionnelle se préoccupe d'une manière permanente de la compétence des diplômés qu'elle met sur le marché du travail. Au cours de la présente décennie, la relève s'ajoute aux autres réalités que les écoles doivent prendre en compte dans leurs programmes de formation.

Depuis plus d'une génération, l'École de bibliothéconomie et des sciences de l'information de l'Université de Montréal a dispensé quatre programmes successifs de maîtrise (1970, 1979, 1989 et 1998), soit en réalité un programme par décennie, bien que des modifications mineures aient été apportées à chacun de ces programmes pendant leur fonctionnement. Nous voulons croire que chacun de ces programmes correspondait à l'évolution de la discipline et de la profession, telle que nous pouvions la décoder et l'interpréter. Dès la première réforme du programme de maitrise, de 1976 à 1979, l'EBSI a d'abord poursuivi des travaux qui ont permis d'évaluer le programme existant au niveau du contenu et des méthodes pédagogiques. Cette évaluation impliquait les étudiants, les diplômés et les employeurs. Il s'agissait ensuite de s'enquérir de l'expérience des meilleurs programmes existant dans le monde afin de l'appliquer ici dans un programme novateur, et surtout d'établir les compétences que devrait posséder un étudiant à la fin de ses études de maîtrise. La question des compétences que doit avoir le diplômé après deux années d'études dans notre discipline est capitale. Ce sont elles qui déterminent le contenu du programme et son articulation au cours des deux années d'études. Bien entendu, on ne peut tout enseigner à l'intérieur de quatre trimestres d'enseignement. Quels sont les domaines vraiment essentiels, d'une part, et les domaines pertinents, mais parfois accessoires ou périphériques, d'autre part? Quelles sont les compétences minimales que doivent posséder les étudiants qui quittent l'EBSI après deux ans de maîtrise? En raison de ces interrogations, depuis 1979, tous les cours qui constituent la première année de maîtrise sont des cours obligatoires. Une décision qui fait consensus parmi les professeurs de l'École depuis 25 ans. Elle ne reçoit pas toujours l'adhésion des diverses cohortes d'étudiants qui voudraient choisir eux-mêmes des cours dès la première année de maîtrise. 
Cette préoccupation, axée sur les compétences que doit maitriser le professionnel de l'information pour entrer dans le monde du travail, a guidé les différentes réformes de programmes qui ont eu cours à l'EBSI. Faisant référence à la dernière réforme qui a mené au programme de maitrise de 1998, Pierrette Bergeron a fait état de la consultation auprès de représentants du marché du travail, soit les employeurs d'alors et les employeurs potentiels de professionnels de l'information (Bergeron 1997). Ces personnes consultées ont noté que le développement du domaine s'articule autour de changements informationnels, organisationnels et sociaux ainsi que de changements technologiques.

"Les changements informationnels sont caractérisés par:

- une attention de plus en plus grande au contenu informationnel (repérage, organisation, validation et diffusion), alors que l'intérêt est présentement davantage sur le contenant, ce qui exigera des professionnels capables de traiter le contenu informationnel et de développer des outils de navigation, de recherche et des interfaces qui soient orientées vers les usagers;

- une connaissance de plus en plus multiforme et distribuée; et

- un décloisonnement des sources et des pratiques dinformation.

Les changements technologiques entraîneront, entre autres;

- l'intégration des technologies et de l'autoroute de l'information;

- le développement d'intranets;

- la multiplication des supports sur lesquels l'information est enregistrée et la complémentarité de ces supports; et

- le développement de la normalisation.» (Bergeron 1997).

Il résulte de ces constats que l'EBSI doit former des spécialistes de la création et de la gestion de l'information. Quant aux connaissances spécifiques dispensées dans le programme d'études, elles sont nombreuseset reposent sur une forte culture générale. Elles portent notamment sur:

- les enjeux économiques, politiques, juridiques, sociaux et technologiques de la société de l'information;

- l'organisation, le traitement, la représentation, le repérage et la diffusion de l'information, des documents et de la connaissance;
- sur les technologies de linformation et des documents électroniques;

- les lois et les normes;

- la gestion des services et des ressources;

- la veille stratégique;

- l'utilisation de l'information et le comportement informationnel des diverses clientèles;

- la formation des utilisateurs.

De plus, les diplômés doivent posséder de nombreuses qualités personnelles (créativité, curiosité, entrepreneurship, empathie, dynamisme, flexibilité) alliées à des qualités de travail (esprit d'analyse et de synthèse, esprit de décision, esprit de service, habileté à la communication, connaissances de langues étrangères). À la lumière de cette énumération, corollaire des études et des consultations d'employeurs, on constate que les attentes sont élevées tant pour les compétences à acquérir que pour les étudiants appelés à les posséder.

Ces constatations, faites à l'occasion de la dernière réforme de programme à l'EBSI et qui appuient le programme dispensé actuellement, rejoignent des constatations observées ailleurs. Car les mutations de la nature du travail des bibliothécaires et des professionnels de l'information sont depuis longtemps exposées et débattues dans la littérature professionnelle. Elles touchent à la fois la description détaillée des éléments du travail professionnel, la nature des emplois vue à travers les affichages de postes, de même que le rôle et les appellations des professionnels de l'information.

Le monde des professionnels de l'information, il faut aussi le dire, est un monde aux contours assez flous. Les sciences de l'information ne sont pas une appellation contrôlée. On peut voir les métiers de la documentation comme un terrain où s'affrontent de plus en plus des diplômés ayant diverses formations et défendant des valeurs différentes (Bouthillier 2002). Parmi les nombreuses compétences recherchées, France Bouthillier en dénote deux qui ressortent nettement: les compétences technologiques et les compétences de gestion. Il est manifeste que les écoles de bibliothéconomie et des sciences de l'information ont mieux réussi, à ce jour, en informatique et en technologies de l'information que dans les compétences de gestion. Or, en cette période de pénurie de gestionnaires dans le monde des bibliothèques et des services d'information, cette carence ressort nettement. C'est sans doute le défi le plus grand auquel sont confrontées actuellement les écoles de bibliothéconomie et des sciences de l'information dans le contexte de la relève.

Il en est de même des conclusions des travaux de l'Alliance of Libraries, Archives and Records Manage- 
Les écoles, dont l'EBSI,

ment (ALARM), qui, au plan canadien, a analysé les compétences requises en archivistique et en bibliothéconomie au cours de la décennie 1990. Sept compétences professionnelles ont été retenues:

- créer/maintenir des programmes, des services;

- acquérir/ disposer des ressources d'information;

- organiser/ décrire des ressources d'information;

- fournir des services de référence, de recherche et de consultation;

- fournir des services d'accès et de soutien;

- fournir des services informatiques;

- entreposer/protéger les ressources d'information.

En outre, le professionnel de l'information doit posséder des compétences générales en démontrant des habiletés de gestion ainsi que des habiletés personnelles et interpersonnelles (Gendron 1999). Les qualités interpersonnelles (orientation client, habileté à la communication, leadership, capacité de travailler en équipe) et personnelles (esprit d'analyse et de synthèse, innovation, sensibilité à la culture organisationnelle, capacité à gérer son développement professionnel) rejoignent les conclusions d'autres études et observations.

Les compétences reconnues comme essentielles à la pratique professionnelle en Amérique du Nord se retrouvent dans les études entreprises ailleurs dans le monde. Dominique Lahary et Dominique Arot les mentionnent dans l'analyse de postes affichés en France (Lahary 1994 et Arot 2000). L'enquête de l'Australien, Michael Middleton, auprès des diplômés en bibliothéconomie, mène à la conclusion que, parmi 189 compétences considérées comme les plus importantes dans la pratique de la profession, les suivantes prédominent nettement:

- la référence traditionnelle et automatisée;

- la qualité de communicateur;

- la formation documentaire;

- la gestion des services et du personnel;

- l'informatique et les technologies de l'information; et

- la capacité pour le professionnel de l'information de développer ses propres compétences et connaissances, tout au long de sa carrière (Middleton, 2003).

On peut dire que les études au plan international comme au plan national font consensus concernant les compétences que doivent posséder aujourd'hui les diplômés des écoles de bibliothéconomie et des sciences de l'information, et, qu'en raison du problème de la relève et du besoin de gestionnaires qui est posé avec acuité, les compétences en gestion ressortent fortement.

\section{L'EMPLOI DES DIPLÔMÉS DE L'EBSI}

Tout particulièrement au cours des années 80 , les écoles de bibliothéconomie et des sciences de l'information agréées par l'American Library Association, c'est-à-dire la grande majorité des écoles américaines et canadiennes, ont été confrontées à une crise qui a amené plusieurs d'entre elles, et non les moindres (Chicago, Columbia, Case Western Reserve notamment), à fermer leurs portes. Ce bouleversement a forcé les autres écoles à s'interroger sur leur mission, leur domaine de formation, le contenu de leur enseignement et les nouvelles réalités du monde du travail en information et documentation. Les écoles, dont l'EBSI, ont ajouté à la bibliothéconomie l'appellation «sciences de l'information ou études en information", élargissant leurs champs d'intérêt aux sciences de l'information et parfois à l'archivistique et développant de nouveaux programmes interdisciplinaires de premier cycle en information-communication, en partenariat avec des facultés de communication, d'éducation ou de management.

Outre la nécessité de survivre, l'objectif essentiel de ces transformations était de conquérir de nouveaux marchés de l'emploi au moment où le marché traditionnel était très faible, voire inexistant. À partir des années 80 , les écoles ont mis résolument l'accent sur les nouveaux marchés de l'information axés sur une expertise en informatique documentaire et en technologies de l'information, de même que sur la gestion de l'information dans l'entreprise. On doit constater que le climat de profond malaise de la décennie 1980 et des débuts de la décennie 1990 s'est en grande partie résorbé. Le passage à la "société de l'information" semble mieux accepté. Le défi des technologies de l'information a été relevé avec succès dans nos écoles. Les domaines de formation ont été élargis à des disciplines-sœurs ou connexes. Contrairement à ce qui se passait, il y a 20 ans, les écoles ont désormais pris en compte les marchés de l'emploi dans l'élaboration de leurs programmes.

La crise qui a affecté les écoles américaines de bibliothéconomie et des sciences de l'information a touché également les écoles canadiennes, mais elle n’a mis en péril l'existence d'aucune d'entre elles, même si plusieurs ont été intégrées à des facultés d'éducation (McGill et Alberta), de communication (Western Ontario) ou de management (Dalhousie). Les sept écoles canadiennes (Toronto, Western Ontario, Alberta, UBC, McGill, Montréal et Dalhousie) ont des 
caractéristiques d'écoles régionales qui visent des marchés particuliers (Curry 2000). On constate que chacune de ces écoles a revu ses orientations et ses programmes et a su se doter d'une spécialité qui la distingue des autres. Pour l'EBSI de l'Université de Montréal, la spécificité a trait à la formation en archivistique et en gestion stratégique de l'information.

$\mathrm{Au}$ cours des 20 dernières années, toutes les écoles ont dû intensifier leur attention vis-à-vis d'un marché de l'emploi en pleine transformation. En 1987, à l'occasion du $25^{\mathrm{e}}$ anniversaire de l'EBSI, Réjean Savard a mené, avec ses étudiants, une enquête visant à mieux connaitre le cheminement de carrière des diplômés, à faire le point sur la situation de l'époque et à prévoir les tendances du marché de l'emploi en information documentaire au Québec (Savard 1987). On constate qu'après 1980 la majorité des emplois obtenus à plein temps sont de type contractuel. Malgré l'état de sous-développement des bibliothèques publiques et des bibliothèques scolaires, les bibliothèques spécialisées sont celles qui embauchent le plus de diplômés. Ces dernières ont des liens avec le secteur privé, et les nouveaux marchés de l'information. Une décennie plus tard, à l'occasion de la dernière réforme du programme de maîtrise, l'École sondait ses diplômés des dernières années (de 1991 à 1995), sur les emplois qu'ils occupent et leur perception du développement du marché du travail (Tabah et Bernhard 1998). Il en ressort que les nouveaux diplômés occupent en majorité des emplois contractuels à plein temps. Le pourcentage d'emplois dans les secteurs non traditionnels est passé de 13,9\% en 1987 à 19,8 \% pour la période 1991-1995. Malgré cette hausse relativement faible, les diplômés 19911995 voient comme domaines d'avenir la consultation et le courtage, l'édition électronique, la veille technologique, la formation des utilisateurs, la création et la gestion des banques de données, la gestion des documents électroniques et des ressources Internet.

En corollaire de l'enquête de 1987 qu'il a conduite auprès des diplômés de l'EBSI, Réjean Savard a poursuivi chaque année ce sondage auprès des diplômés de chaque promotion en leur envoyant un questionnaire neuf mois après leur départ de l'École, de façon à faire le point sur l'emploi. Avaient-ils décroché un emploi? Quel genre d'emploi et combien de temps avaient-ils mis pour l'obtenir? Les résultats de ces sondages paraissent dans la plupart des numéros annuels d'En direct de l'EBSI.

Ainsi entre 1990 et 2002, près du tiers des diplômés de l'École ont trouvé un emploi dans les bibliothèques spécialisées et les centres de documentation. Les bibliothèques publiques constituent le deuxième marché significatif, avec des proportions qui varient entre $32 \%$ en 1998 et $15 \%$ en 2002 . Ce n'est quà partir de 2001 que les bibliothèques universitaires et les bibliothèques nationales se sont révélées des employeurs importants (entre 12 et $22 \%$ du marché). Une donnée demeure constante tout au long des années couvertes par les enquêtes de Réjean Savard: environ $90 \%$ des diplômés ont décroché un emploi au cours des six mois qui ont suivi la fin de leurs études universitaires. Quant à la nature de ces emplois, ce sont en grande majorité des contrats annuels renouvelables (de $35 \%$ en 1998 à $77 \%$ en 2000). Depuis la cohorte des diplômés de 2001, les emplois contractuels semblent en baisse, sans que l'on puisse à ce jour déceler une tendance nette $(25 \%$ en 2001 et $47 \%$ en 2002). Il faut noter, par ailleurs, que les emplois contractuels, renouvelables pour la plupart, constituent des étapes d'obtention d'un emploi régulier à plein temps, et il semble bien que le renouvellement du contrat fait dorénavant partie de la nouvelle donne pour l'obtention d'un emploi régulier. Toutes les questions liées à l'emploi de leurs diplômés intéressent au premier chef les écoles de bibliothéconomie et des sciences de l'information en général, et l'EBSI, en particulier, en raison des spécificités du marché de l'emploi pour les diplômés québécois de langue française. Il est capital pour l'EBSI de faire du suivi du marché de l'emploi un indicateur permanent, autant pour des études longitudinales sur le sujet que pour orienter le contenu du programme de maîtrise professionnelle.

\section{CONCLUSION}

À l'instar des autres écoles de bibliothéconomie et des sciences de l'information du continent, l'EBSI a dû faire face depuis près d'un quart de siècle à un double défi; la transformation de la discipline de la bibliothéconomie et les mutations du marché de l'emploi. Elle a répondu à ces défis en réformant profondément ses programmes d'études grâce à une méthodologie rigoureuse et à un élargissement des disciplines enseignées telles que les sciences de l'information et l'archivistique. Elle a, en outre, accordé une attention considérable à l'examen de l'emploi de ses diplômés au cours de ces années. En ce début du présent siècle, l'on peut prétendre d'une manière réaliste, que relever ces défis aura été bénéfique pour l'EBSI. En effet, ils ont été des facteurs de renouvellement et de développement. Forte de ces expériences, l'École est prête à faire face aux futures modifications ou mutations des disciplines ou des professions de l'information, grâce aux programmes d'étude qu'elle a mis sur pied pour les trois 
cycles universitaires et aux nombreuses recherches que mènent ses professeurs.

Une attention soutenue sur les compétences disciplinaires et professionnelles, et un examen minutieux du marché de l'emploi continueront à faire partie des préoccupations constantes de l'École. Elles sont des bornes qui lui indiquent qu'elle est dans la bonne direction, que la formation dispensée à ses étudiants est pertinente, et que ces derniers sont tout à fait capables, au terme de leurs études et en dépit d'un marché du travail souvent instable, de relever les exigences des nouveaux emplois de la société de l'information.

Pour sa part, la relève se présente comme une nouvelle réalité du monde des professionnels de l'information pour les prochaines dix années. Il s'agit de remplacer une portion importante des professionnels dans les bibliothèques universitaires surtout, mais aussi dans d'autres types de bibliothèques, dans les services documentaires ou dans les centres d'archives. La relève dans ces domaines sera couplée avec les besoins de personnel professionnel d'autres domaines, tels que les bibliothèques publiques. Celles-ci devraient se développer au cours des prochaines années, car avec environ 300 professionnels (quand ils sont plus de 1000 en Ontario!), elles sont bien loin d'avoir le personnel professionnel requis pour une politique de lecture publique de qualité. Il en est de même dans le secteur des archives qui s'est imposé comme un secteur dynamique de la gestion de l'information tant dans l'entreprise que dans toute organisation de la société contemporaine. Pour relever ce stimulant défi de la relève, l'EBSI devra être à l'écoute de ces nouveaux besoins et travailler en étroite relation avec les milieux professionnels confrontés au même problème, afin d'adopter les ajustements adéquats face à ce nouveau contexte. $\diamond$

\section{SOURCES CONSULTÉES}

Anderson, Janice et al. 2003. Evolution and survival of the fittest in library and information services. Information Outlook, vol. 7, $\mathrm{n}^{\circ} 2$ (February) : 20-24.

Arot, Dominique. 2000. Les valeurs professionnelles du bibliothécaire, Bulletin des bibliothèques de France vol. 45, $\mathrm{n}^{\circ} 1$ : 33-41.

Association des universités et des collèges du Canada. 2002. Orientations. Le milieu universitaire. Ottawa. $94 \mathrm{p}$.

Bergeron, Pierrette. 1997. Quelles compétences devra maîtriser le professionnel de l'information pour pénétrer le marché du travail de demain? Analyse de la perception des représentants du marché du travail. Argus vol. 19, n 1 (printemps-été): 21-26.

Bertrand-Gastaldy, Suzanne et Marcel Lajeunesse. 1990. La réaction de l'École de bibliothéconomie et des sciences de l'information de l'Université de Montréal aux mutations de la société de l'information: l'évaluation et la révision de son programme de maitrise. Argus vol. 19, n 1 (printemps): 11-24.

Bouthillier, France. 2002. La concurrence dans les métiers de la documentation: réalité ou fiction. Bulletin des bibliothèques de France vol. 47, n 1 : 36-41.

Conférence des recteurs et des principaux des universités du Québec (CRÉPUQ). Statistiques générales des bibliothèques universitaires québécoises, annuel.

Curran, William. 2002. La relève: à qui le tour? Argus vol. $31, \mathrm{n}^{\circ} 2$ (automne) : 33-37.

Curry, Ann. 2002. Canadian LIS education: trends and issues. Education for information vol. 18: 325-337.

En direct de l'EBSI, $\mathrm{n}^{\circ} 1$ (1988)

Gendron, Céline. 1999. Profil des compétences des spécialistes en gestion des ressources informationnelles: une analyse intégrée et intersectorielle des compétences requises dans le domaine des archives, de la bibliothéconomie et de la gestion documentaire. Argus vol. 28, n³ (hiver): 11-19.

Hernon, Peter, Ronald R. Powell and Arthur P. Young. 2001. University library directors in the Association of Research Libraries: the next generation, part one. College and Research Libraries vol. $62, \mathrm{n}^{\circ} 2$ (March): 116-145.

Lahary, Dominique. 1994. Du profil du poste au métier. Bulletin d'informations de l'Association des bibliothécaires français $n^{\circ} 164$ ( $3^{\mathrm{e}}$ trimestre): 71-82.

Law, Margaret. 2000. Age demographics and libraries. Feliciter $\mathrm{n}^{\circ} 5$ : 263-266.

Middleton, Michael. 2003. Skills expectations of library graduates. New Library World vol. 104, ${ }^{\text {os }} 1184 / 1185$ : 42-56.

Reicher, Daniel et Marcel Lajeunesse. 1979. Le nouveau programme de maitrise à l'École de bibliothéconomie: génèse et organisation. Argus vol. 8, (mai-août): 43-48.

Savard, Réjean. 2000. La formation des bibliothécaires en Amérique du Nord; modernité et valeurs professionnelles. Bulletin des bibliothèques de France vol. 45, nº 1 : 42-49.

Savard, Réjean et al. 1987. Enquête sur les diplômés de l'EBSI. Argus vol. $16, \mathrm{n}^{\circ} 3$ (septembre) : 81-87.

Tabah, Albert et Paulette Bernhard.1998. Emplois occupés après la diplômation et champs d'activité perçus comme importants « aujourd'hui et dans cinq ans » selon l'enquête menée auprès des diplômés de l'EBSI (1991-1995), Argus vol. 27, n² (automne): 5-13. 